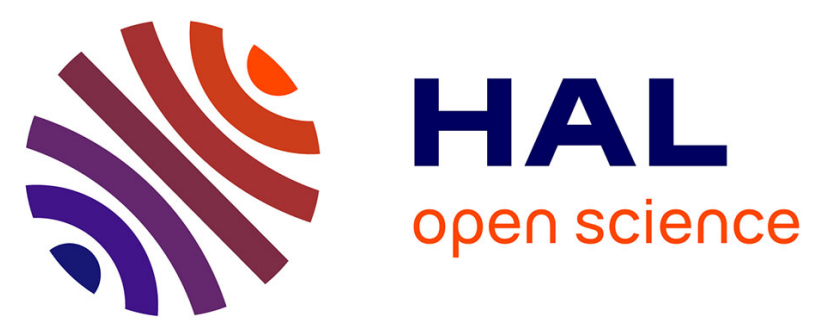

\title{
Control of power converters with hybrid affine models and pulse-width modulated inputs
}

Carolina Albea-Sanchez, Antonino Sferlazza, Fabio Gómez-Estern, Francisco

Gordillo

\section{- To cite this version:}

Carolina Albea-Sanchez, Antonino Sferlazza, Fabio Gómez-Estern, Francisco Gordillo. Control of power converters with hybrid affine models and pulse-width modulated inputs. IEEE Transactions on Circuits and Systems I: Regular Papers, 2021, 68 (8), pp.3485 - 3494. 10.1109/TCSI.2021.3083900 . hal-03326994

\section{HAL Id: hal-03326994 \\ https://hal.laas.fr/hal-03326994}

Submitted on 26 Aug 2021

HAL is a multi-disciplinary open access archive for the deposit and dissemination of scientific research documents, whether they are published or not. The documents may come from teaching and research institutions in France or abroad, or from public or private research centers.
L'archive ouverte pluridisciplinaire HAL, est destinée au dépôt et à la diffusion de documents scientifiques de niveau recherche, publiés ou non, émanant des établissements d'enseignement et de recherche français ou étrangers, des laboratoires publics ou privés. 


\title{
Control of power converters with hybrid affine models and pulse-width modulated inputs
}

\author{
Carolina Albea, Antonino Sferlazza, Francisco Gordillo, Fabio Gómez-Estern
}

\begin{abstract}
In this paper, hybrid dynamical systems theory is applied to the analysis and control of switched converters with Pulse-Width Modulated (PWM) inputs. The system is described by a state-space model with continuous flows and discrete jumps, without averaged equations. The modulation effects are captured in full without using time-dependent signals, by enlarging the state vector to include the PWM waveform generation process. Furthermore, the sample-and-hold mechanism associated with the sampling frequency is also taken into account with this approach. A control law is proposed based on a Lyapunov function candidate. Furthermore, convergence sets and the steady state jitter, inherent to PWM-based controllers, are analyzed estimating limit sets for the augmented state. Consequently, output chattering can be bounded. By using hybrid dynamical system theory, the control designer gains a deeper understanding of the effect of modulation in the closedloop dynamics, avoiding the problems associated with the use averaged models. Experimental results validate the proposed method.
\end{abstract}

Index Terms - Converter control, PWM, hybrid dynamical system, Lyapunov analysis.

\section{INTRODUCTION}

In electronic power converters, the output of the controller is usually a discrete signal connected to the transistor gates with the aim of driving the output to some desired value. Usually, the design of controllers for this type of systems is based on averaged models that consider the control inputs as continuous signals [1-4]. In order to implement the resulting controller, the computed control signal is discretized using a modulator. The most common modulators use Pulse-Width Modulation $[5,6]$. This approach may limit the accuracy of the signals in steady state [7] and even can fail to predict the system behavior

This work has been partially funded by the Agence Nationale de la Recherche (ANR)-France under Grant ANR-18CE40-0022-01, by the Agencia Estatal de Investigación (AEI)Spain under Grant PID2019-105890RJ-100/10.13039/100014440, PID2019-109071RB-I00/10.13039/100014440, DPI2016-75294-C2-2R10.13039/100014440 by Junta de Andalucía Programa Operativo FEDER Andalucía 2014-2020, US-1264655 and by the ECSEL joint Undettaking, under Grant No. 783158.

This work has been conducted while C. Albea was with CNRS, LAAS, Université de Toulouse, 7 avenue du colonel Roche, F-31400 Toulouse, France. Now, C. Albea is with Universidad de Sevilla, Department of System Engineering and Automatic Control, Camino de los Descubrimientos s/n, 41092, Seville, Spain (e-mail: calbea@us.es).

A. Sferlazza is with University of Palermo, Department of Engineering, viale delle scienze Ed. 10, 90128 Palermo, Italy. E-mail: antonino.sferlazza@unipa.it.

F. Gordillo is with Universidad de Sevilla, Department of System Engineering and Automatic Control, Camino de los Descubrimientos s/n, 41092, Seville, Spain (e-mail: gordillo@us.es).

F. Gómez-Estern is with Universidad de Loyola Andalucía, Av. de las Universidades, s/n, 41704 Dos Hermanas, Seville, Spain (e-mail: fgestern@uloyola.es).
[8]. Besides, it is usual to ignore the discrete-time nature of the control signals and the sample-and-hold mechanism.

Normally, PWM-based controllers [3,4] are commonly based on a separation principle: a high-level control algorithm, usually based on a simple model, computes a continuous signal that is converted into a discrete input in the PWM, low-level block. In order for this separation principle to be tractable, the high-level controller is designed based on a model of the plant together with the modulator block that is simplified to keep the linear timeinvariance properties. This implies averaging or approximating the exponential matrices by linear expressions, and generally neglecting the specifics of the PWM waveform. The approach works well provided that sampling rates are high. However, it is known that PWM blocks have an effect on the output, $[4,9]$. PWM modulation of analogue control signals limits in some ways the degrees of freedom of the control action, as signal values are discrete, and they can only be changed once or a few limited times along the sampling interval. This effect is often ignored, but at a micro scale the signal mismatch causes output jitter in steady state. Nevertheless, a modulator in the system model introduces the challenge of dealing with time-dependent signals and, consequently, non-autonomous dynamics.

There exist control techniques that avoid the use of averaged models and deal directly with the discrete nature of signals, such as sliding mode control [10], model predictive control $[11,12]$. Nevertheless, dealing directly with discrete input signals, avoiding the use of PWM gives rise to the loss of an important degree of freedom of the PWM mechanism. With PWM, the switches can be ordered at any instant inside the sampling interval (with a limitation in the total number of switches in this interval); with most of the techniques that avoid the use of averaged models, the control input can only be changed at the sampling instants. This makes it necessary to increase the sampling frequency. A solution was presented in [13] with a high number of LMI conditions to be satisfied, dealing to a conservative result.

Hybrid Dynamical System (HDS) theory can help in building autonomous state-space models of PWM-based switched converters, and also to design suitable controllers. Indeed, HDS theory is a mature framework for analyzing dynamical behaviours made of continuous dynamic fields (flows) and discrete changes in signals (jumps). Following notorious results from the literature $[14,15]$, it is easy to ascertain the conditions for an autonomous hybrid system to have proper solutions, to avoid conflictive behaviours such as Zeno dynamics, and to ensure stability and steady-state specifications. For relevant results that 
will be used here, see also [16-18] In this context, the authors in [19] proved that, for general systems under some assumptions, that the solutions of an averaged system are suited approximations of the original one, mentioning power converters as application. Nevertheless, these hybrid approaches suffer from the same drawback than other techniques mentioned above: the discrete control input can only be changed at the sampling instants.

Switched power systems often appear in the hybrid framework as affine systems. This class of systems is described by standard state-space equations, where matrices $A$ and $B$ can change depending on the state of the switches, see $[20,21]$. There exist several results about controlling switched affine systems without considering PWM. For instance, robust and input-to-state stability has been analyzed in [22]. In [23], and more recently in $[24,25]$, this framework is presented and applied to DCDC converters. The main feature in these references is the implementation of an aperiodic sampled-data control signal with arbitrarily fast switching, making possible the appearance of Zeno behaviour. This practical difficulty has been overcome in [26].

This paper aims at designing a control law for power converters with affine models using hybrid dynamical systems theory. An advantage of this approach is to provide a rigorous state-space autonomous model that encompasses all elements of the converter architecture: power converter, PWM and sample-and-hold mechanism. Some results in this paper require sufficiently small sampling intervals, but this is not an a priori condition for modeling; it only arises when computing the convergence set, and the relation between chattering and the PWM-sampling period is made explicit.

The novel idea developed here is focused on power converters with only two functioning modes, which are very common in power electronics [27-29]. A new controller is proposed to stabilize a limit cycle, based on a rigorous model, different from the classical averaged models used in the literature. The stability design is obtained at the cost of a harder analysis compared with usual controllers based on averaged models, but the controller implementation does not grow in complexity, and it can be readily adapted to other modulation techniques. Furthermore, an exact estimation is provided for the chattering in steadystate. Finally, experimental results show satisfactory performance of the proposed control loop.

This paper is organized as follows. The problem statement is given in Section II. The hybrid general model of switched PWM-based converters is presented in Section III. In Section IV, the main result is presented. Section V and Section VI present simulation and experimental results, respectively. Finally, the paper closes with a conclusion section.

Notation: Throughout the paper $\mathbb{N}$ denotes the set of the natural numbers, $\{0,1,2,3, \ldots\}$, and $\mathbb{R}$ the set of real numbers, $\mathbb{R}^{n}$ the $n$-dimensional Euclidean space and $\mathbb{R}^{n \times m}$ the set of all real $n \times m$ matrices. The set of non-negative real numbers is denoted by $\mathbb{R}_{\geq 0} . M \succ 0$ (resp. $M \prec 0$ ) represents that $M$ is a symmetric positive (resp. negative) definite matrix. 0 is a zero matrix of suited dimensions. $\mathrm{He}(M)$ is the hermitian matrix of $M$, i.e. $\left(M+M^{\top}\right)$. Finally, $\operatorname{sat}_{a}^{b}(\phi)$ is the standard saturation function defined in $\mathbb{R} \mapsto[a, b]$.

\section{Problem statement}

Many switched power systems made of linear components and switching devices can be modelled as switched affine systems, as follows

$$
\dot{z}=A_{\sigma} z+\mathcal{B}_{\sigma},
$$

where $z \in \mathbb{R}^{n}$ is the vector of physical state variables, i.e. voltages and currents and $\sigma \in\{0,1,2, \ldots, N-1\}$ is the control input that represents the functioning mode of the converter according to the switching state. Finally, $A_{\sigma}$ and $\mathcal{B}_{\sigma}$ are matrices of suitable dimensions.

Model (1) covers many applications of power converters, such as the buck converter, the boost converter, the quadratic boost converter, the half bridge converter, the boost inverter, etc. In this paper, only two functioning modes, $N=2$, are considered.

These systems are generally controlled by continuoustime control inputs, i.e., $\sigma \in\{0,1\}$ is modelled by a continuous signal $\lambda \in[0,1]$, obtained via averaging techinques, and implemented in (1) by Pulse Width Modulation (PWM), as depicted in Fig. 1. In this block diagram, $x:=z-z_{e}, z_{e}$ is the desired equilibrium point and $\kappa(x)$ represents a continuous control law saturated within the interval $[0,1]$. Furthermore, the control law is usually implemented in a digital device in discrete time by sampling the state of the converter $z(t)$ periodically. Thus, the value of $\lambda$ in Fig. 1 is constant during each sampling interval. The PWM mechanism with a sawtooth carrier is illustrated in Fig.2.

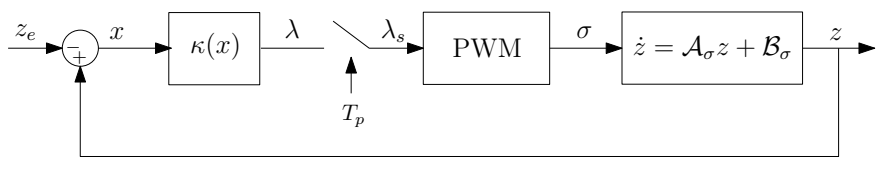

Fig. 1. Feedback scheme.

The control objective is to design a function $\lambda=\kappa(x)$ : $\mathbb{R}^{n} \mapsto \mathbb{R}$ such that the modulated control signal, $\sigma(\lambda)$, ensures the convergence of $z$ to a given operating point $z_{e}$. Due to the sampling mechanism, asymptotic convergence to $z_{e}$ is not possible, and a chattering phenomenon is unavoidable. In any case, the desired operating point $z_{e}$ must satisfy the following assumption.

Assumption 1: Given an operating point $z_{e}$ there exists a $\lambda=\lambda_{e} \in[0,1]$ such that the following convex combinations holds,

$$
0=\left(A_{0}+\left(A_{1}-A_{0}\right) \lambda_{e}\right) z_{e}+\mathcal{B}_{0}+\left(\mathcal{B}_{1}-\mathcal{B}_{0}\right) \lambda_{e} .
$$

This standard assumption for switched affine systems ([30, 31] guarantees the existence of a switched signal for system 


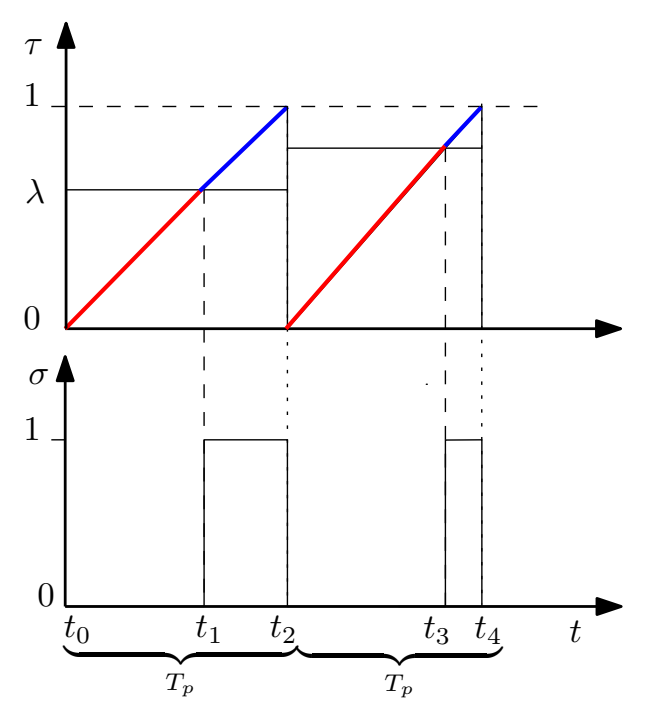

Fig. 2. PWM mechanism with sawtooth carrier. Top: $T_{p}$-periodic sawtooth carrier, $\tau$, and $\lambda$ the duty cycle. Bottom: output of the PWM block, $\sigma$.

(1), inducing an equilibrium in $z=z_{e}$ in the generalized sense of Krasovskii. This means that, in steady state, $\sigma$ is expected to be a periodic signal of period $T_{p}$, spending a time $\lambda T_{p}$ in mode 1 and $(1-\lambda) T_{p}$ in mode 0 , corresponding to the convex combination of the right hand of (2). Then, the time spent in each mode will be distributed in every time period according to the modulator shown in Fig 2. This regularization allows to identify the duty cycle, $\lambda$ as the control signal.

In this paper, it is assumed without loss of generality that a carrier sawtooth waveform period starts at $t=0$, as well as a sampling period .

The error equation associated with (1) in each sampling time $t_{k}$ for all $k \in \mathbb{N}$ can be written as:

$$
\dot{x}=A_{\sigma} x+B_{\sigma},
$$

where $B_{\sigma}:=\mathcal{B}_{\sigma}+A_{\sigma} z_{e}$ such that $B_{\lambda_{e}}=0$.

Direct integration of the dynamics of $x$ in (3) along the sawtooth carrier period (Fig. 2), starting from an initial condition $x\left(t_{2 k}\right)$ at $t=t_{2 k}$ yields

$$
\begin{aligned}
& x\left(t_{2 k+1}\right)=e^{A_{0} \lambda T_{p}} x\left(t_{2 k}\right)+\left(e^{A_{0} \lambda T_{p}}-I\right) A_{0}^{-1} B_{0} \\
& x\left(t_{2 k+2}\right)=e^{A_{1}(1-\lambda) T_{p}} x\left(t_{2 k+1}\right)+\left(e^{A_{1}(1-\lambda) T_{p}}-I\right) A_{1}^{-1} B_{1} .
\end{aligned}
$$

being $t_{2 k+2}-t_{2 k}=T_{p}$ for all $k \in \mathbb{N}$.

The desired behavior in steady state corresponds to $\lambda=$ $\lambda_{e}$ and a limit cycle for $x$ (see Fig. 3 ) considered in the following property.

Property 1: Consider a couple $(x, \lambda)=\left(x_{e}, \lambda_{e}\right)$ associated to (3) that satisfies Assumption 1. If there exist two vectors $x_{e, T_{p}}$ and $x_{e, \lambda_{e} T_{p}}$ fulfilling

$$
\left\{\begin{array}{l}
x_{e, \lambda_{e} T_{p}}=e^{A_{0} \lambda_{e} T_{p}} x_{e, T_{p}}+\left(e^{A_{0} \lambda_{e} T_{p}}-I\right) A_{0}^{-1} \mathcal{B}_{0} \\
x_{e, T_{p}}=e^{A_{1}\left(1-\lambda_{e}\right) T_{p}} x_{e, \lambda_{e} T_{p}}+\left(e^{A_{1}\left(1-\lambda_{e}\right) T_{p}}-I\right) A_{1}^{-1} \mathcal{B}_{1},
\end{array}\right.
$$

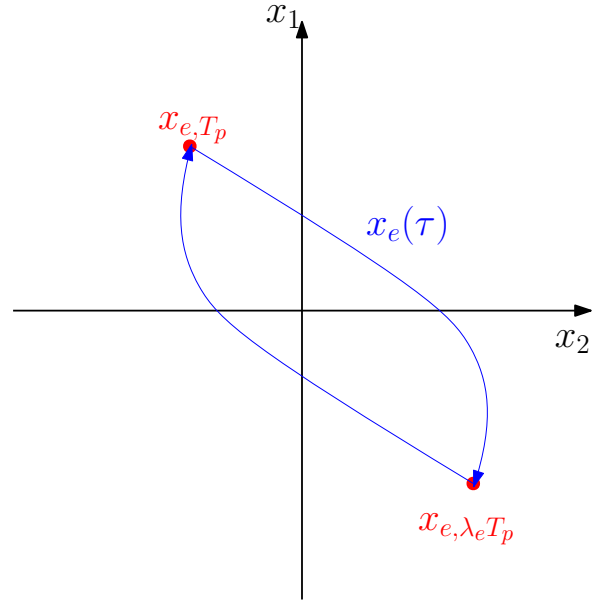

Fig. 3. Example of limit cycle according to Property 1 for a case with $n=2$. The red points and the blue trajectory represent points (6) and curve (7), respectively.

then, there exists a limit cycle

$$
\chi=\left\{\left(x \in \mathbb{R}^{n} / \exists \tau \in\left[0, T_{p}\right): x=x_{e}(\tau)\right\}\right.
$$

in (3) defined by

$$
\left\{\begin{array}{c}
x_{e}(\tau)=e^{A_{0} \lambda_{e} \tau} x_{e, T_{p}}+\int_{0}^{\tau} e^{A_{0} \lambda_{e} \bar{\tau}} \mathcal{B}_{0} d \bar{\tau} \\
\text { if } 0 \leq \tau<\lambda_{e} T_{p} \\
x_{e}(\tau)=e^{A_{1} \lambda_{e}\left(\tau-\lambda_{e} T_{p}\right)} x_{e, \lambda_{e} T_{p}}+\int_{\lambda_{e} T_{p}}^{\tau} e^{A_{1} \lambda_{e}\left(\bar{\tau}-\lambda_{e} T_{p}\right)} \mathcal{B}_{1} d \bar{\tau} \\
\text { if } \quad \lambda_{e} T_{p} \leq \tau<T_{p}
\end{array}\right.
$$

Proof. Curve (7) is a closed and isolated trajectory associated to (3). It is isolated because $\left(x_{e}, \lambda_{e}\right)$ and (6) form a linear system of two equations with unknowns $x_{e, T_{p}}$ and $x_{e, \lambda_{e} T_{p}}$ and then, (7) is unique. Consequently, (7) is a limit cycle.

Then, we are in conditions of formulating the problem.

Problem 1: Consider the switched system (1) with $N=2$ and a PWM with a sawtooth carrier, as shown in Fig. 2. Then, the objectives here are

- to model the closed-loop system considering its hybrid character, that is, the existence of both discrete-time and continuous-time signals, as well as the sample and hold mechanism with a given periodic sampling time $T_{p}$.

- To design a control law for the duty cycle $\lambda$, without using averaged models.

- To achieve convergence of $x(t)$ to limit cycle (7) and to analyze stability properties for the hybrid system.

\section{HYBRID DYNAMICAL MODEL}

In this section, we will use the framework given in [14] about hybrid dynamical systems to model the controlled system, considering continuous-time and discrete-time dynamics. Hence, we propose the following hybrid dynamical 
model of the controlled switched system (3), considering a sawtooth carrier for the PWM mechanism,

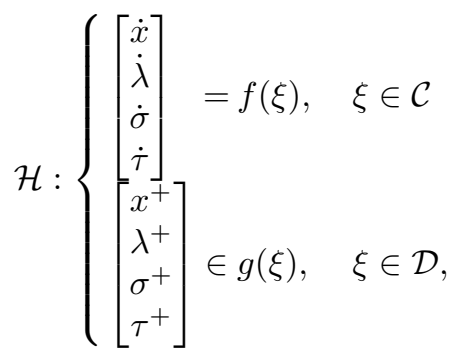

where $\xi=\left[\begin{array}{llll}x & \lambda & \sigma & \tau\end{array}\right]^{\top} \in \mathbb{H}$ such that $\mathbb{H}:=\mathbb{R}^{n} \times$ $[0,1] \times\{0,1\} \times\left[0, T_{p}\right]$. The maps $f$ and $g$ capture both the continuous-time and discrete-time dynamics and are defined as follows:

$$
f(\xi)=\left[\begin{array}{c}
A_{\sigma} x+B_{\sigma} \\
0 \\
0 \\
1
\end{array}\right],
$$

The so-called flow and jump sets are

$$
\begin{aligned}
\mathcal{C}_{0} & :=\left\{\xi \in \mathbb{H}: \tau \in\left[0, \lambda T_{p}\right], \sigma=0\right\} \\
\mathcal{D}_{0} & :=\left\{\xi \in \mathbb{H}: \tau=\lambda T_{p}, \sigma=0\right\} \\
\mathcal{C}_{1} & :=\left\{\xi \in \mathbb{H}: \tau \in\left[\lambda T_{p}, T_{p}\right], \sigma=1\right\} \\
\mathcal{D}_{1}: & =\left\{\xi \in \mathbb{H}: \tau=T_{p}, \sigma=1\right\},
\end{aligned}
$$

being

$$
\begin{aligned}
\mathcal{C} & :=\mathcal{C}_{0} \cup \mathcal{C}_{1} \\
\mathcal{D} & :=\mathcal{D}_{0} \cup \mathcal{D}_{1} .
\end{aligned}
$$

This hybrid scheme gathers the complete dynamics of the system. Indeed, the continuous-time dynamics of $x$ evolves according the switching of $\sigma$. The selection of the last one is given by a sawtooth modulator defined by $\lambda$, $\tau$ and $T_{p}$. Variable $\lambda \in[0,1]$ is the duty cycle and $\tau$ is a timer that defines a continuous-time sawtooth signal (the carrier shown in Fig. 2); Finally, $\kappa(x)$ is a function that computes the value of signal $\lambda$ in every sampling time, $t_{2 k}$. Indeed, when the solutions are in the jump set, two things can happen: either $\lambda$ is updated (because the solution is in $\mathcal{D}_{1}$ ), or $\lambda$ does not change (because the solution is in $\left.\mathcal{D}_{0}\right)$.

Solutions to $\mathcal{H}(f, G, \mathcal{C}, \mathcal{D})$ are given on the so-called hybrid time domain: $\operatorname{dom}(\xi) \subset \mathbb{R}_{\geq 0} \times \mathbb{N}$, such that,

$$
\operatorname{dom}(\xi)=\bigcup_{j=0}^{\bar{j}-1}\left(\left[t_{j}, t_{j+1}\right], j\right),
$$

for some sequence $0=t_{0} \leq t_{1} \leq t_{2} \leq \ldots \leq t_{\bar{j}}$ with $\bar{j}$ finite or infinite.

In the next section, a control law will be designed for system (8)-(11), achieving closed-loop stability. Now, let us rewrite the continuous- and discrete-time dynamics of $x$ to establish a Lyapunov function candidate. To do so, define $\Gamma_{\sigma}=\left[\begin{array}{cc}A_{\sigma} & B_{\sigma} \\ 0_{1, n} & 0\end{array}\right]$ such that during the flows:

$$
\frac{d}{d t}\left[\begin{array}{l}
x \\
1
\end{array}\right]=\Gamma_{\sigma}\left[\begin{array}{l}
x \\
1
\end{array}\right] .
$$

Now, the following Lyapunov function candidate is considered

$$
V(x, \lambda, \sigma, \tau)=\max \left\{W(x, \lambda, \sigma, \tau)-\alpha_{1}, 0\right\},
$$

where $\alpha_{1}$ is a positive parameter and $W$ is a quadratic function of $x$, which is defined as follows,

$$
W(x, \lambda, \sigma, \tau):=\left[\begin{array}{c}
x \\
1
\end{array}\right]^{\top} \mathcal{P}_{\sigma}(\lambda, \tau)\left[\begin{array}{l}
x \\
1
\end{array}\right]
$$

with

$$
\begin{aligned}
& \mathcal{P}_{0}(\lambda, \tau):=e^{-\Gamma_{0}^{\top} \tau} \bar{P} e^{-\Gamma_{0} \tau} \\
& \mathcal{P}_{1}(\lambda, \tau):=e^{\Gamma_{1}^{\top}\left(\lambda T_{p}-\tau\right)} e^{-\Gamma_{0}^{\top} \lambda T_{p}} \bar{P} e^{-\Gamma_{0} \lambda T_{p}} e^{\Gamma_{1}\left(\lambda T_{p}-\tau\right)}
\end{aligned}
$$

and

$$
\bar{P}:=\left[\begin{array}{ll}
P & 0 \\
0 & 0
\end{array}\right]
$$

where $P \succ 0 \in \mathbb{R}^{n}$ will be particularized later.

We are in position to define the target compact set, for which uniform globally asymptotic stability (UGAS) will be established:

$$
\mathcal{A}:=\{\xi \in \mathbb{H}: V(x, \lambda, \sigma, \tau)=0\} .
$$

It is worth noting that the Lyapunov function candidate is a single-valued function, it is continuous in $\xi \in \mathbb{H}$ and enjoys nice properties. The motivation for this choice is to have a Lyapunov function that remains constant during flows, keeps unchanged in the jump instants $\left(\left(\frac{j}{2}+\lambda_{j}\right) T_{p}, j\right)$ for $j=2 k, k \in \mathbb{N}$ and gets $\Delta V<0$ in each jump instant $\left(\left(\frac{j+1}{2}\right) T_{p}, j\right)$ for $j=2 k+1, k \in \mathbb{N}$. This makes the candidate Lyapunov function non increasing (decreasing every two jumps), and proves the convergence of state to the set $\mathcal{A}$ after each jump for $j=2 k+1, k \in \mathbb{N}$, and $x$ to the interior of $x^{\top} P x \leq \alpha_{1}$ as $j$ goes to infinity.

\section{MAIN RESUlT}

Inspired by [32], this section presents a controller for the hybrid system (8)-(11) providing uniform global asymptotic stability (UGAS) of the compact set $\mathcal{A}$.

Theorem 1: Consider there is a $\lambda_{e}$ associated with the operating point $x_{e}$ such that Assumption 1 is satisfied, and matrices $P, Q \succ 0 \in \mathbb{R}^{n \times n}$ such that $Q \succ P$ and $M \prec Q-P \in \mathbb{R}^{n \times n}$, satisfying the following conditions

$$
\begin{aligned}
& A_{0}^{\top} P+P A_{0}+\alpha_{2} I \prec-Q \\
& A_{1}^{\top} P+P A_{1}+\alpha_{2} I \prec-Q
\end{aligned}
$$

with $\alpha_{2}>0$ a design parameter that will determine the size of the convergence set, and as will be shown below, is related to the sampling time. Moreover, consider system (8)-(11), with

$$
\kappa(x) \in\left\{\begin{array}{ccc}
\lambda_{e}\left(1-\frac{x^{\top} M x}{2 B_{1}^{\top} P x}\right) & \text { if } & B_{1}^{\top} P x \neq 0 \\
{[0,1]} & \text { if } & B_{1}^{\top} P x=0,
\end{array}\right.
$$


Then, there is a value $T_{p}^{*}\left(\alpha_{1}, \alpha_{2}\right)$ such that for any sampling time $T_{p}<T_{p}^{*}$, the following statements hold

(i) $\mathcal{A}$ is UGAS,

(ii) the set

$$
\mathcal{L}:=\left\{\xi \in \mathbb{H}: x=x_{e}(\tau), \lambda=\lambda_{e}\right\}
$$

is in the interior of $\mathcal{A}$.

Proof.

Hybrid system $\mathcal{H}(f, g, \mathcal{C}, \mathcal{D})$ with function (19) is wellposed because it verifies:

- $\mathcal{C}$ and $\mathcal{D}$ are closed sets in $\mathbb{H}$.

- $f$ is a continuous function, hence it is locally bounded and outer semi-continuous. Moreover, it is convex for each $\xi \in \mathcal{C}$.

- $g$ is outer semi-continuous and locally bounded.

We will consider the proof item by item.

Proof of (i): The proof of this item proceeds applying $[33$, Theorem 1$]$. Note that the candidate Lyapunov function, $V(x, \lambda, \sigma, \tau)(14)$ is continuous in $\mathcal{C} \cup \mathcal{D}_{p}$ and locally Lipschitz near each point in $\mathcal{C} \backslash \mathcal{A}$. Moreover, $V(x, \lambda, \sigma, \tau)$ is strictly positive definite in $(\mathcal{C} \cup \mathcal{D}) \backslash \mathcal{A}$ and radially unbounded. Likewise, it verifies, by definition $V(x, \lambda, \sigma, \tau)=$ 0 , for all $(x, \lambda, \sigma, \tau)$ in $\mathcal{A}$.

The next step of the proof is to ensure that the derivative of $V$ along flows outside of $\mathcal{A}$ is nonpositive (or more precisely in this case, equal to zero). More formally, the objective is to show that

$$
\langle\nabla V(\xi), f(x, \sigma)\rangle \leq 0, \quad \forall(x, \lambda, \sigma, \tau) \in \mathcal{C} \backslash \mathcal{A} .
$$

It is clear, from its definition, that $V(x, \lambda, \sigma, \tau)=$ $W(x, \lambda, \sigma, \tau)-\alpha_{1}$ getting

$$
\begin{aligned}
& \langle\nabla V(x, \lambda, \sigma, \tau), f(x, \sigma)\rangle \\
& =\left[\begin{array}{l}
x \\
1
\end{array}\right]\left(^{\top} \dot{\tau} \frac{\partial}{\partial \tau} \mathcal{P}_{\sigma}(\lambda, \tau)+\dot{\lambda} \frac{\partial}{\partial \lambda} \mathcal{P}_{\sigma}(\lambda, \tau)+\dot{\sigma} \frac{\partial}{\partial \sigma} \mathcal{P}_{\sigma}(\lambda, \tau)\right)\left[\begin{array}{l}
x \\
1
\end{array}\right] \\
& +2\left[\begin{array}{l}
x \\
1
\end{array}\right]^{\top} \mathcal{P}_{\sigma}(\lambda, \tau)\left[\begin{array}{l}
\dot{x} \\
0
\end{array}\right] \\
& =\left[\begin{array}{l}
x \\
1
\end{array}\right]^{\top}\left(\frac{\partial}{\partial \tau} \mathcal{P}_{\sigma}(\lambda, \tau)+\mathcal{P}_{\sigma}(\lambda, \tau) \operatorname{He}\left(\Gamma_{\sigma}\right)\right)\left[\begin{array}{l}
x \\
1
\end{array}\right]=0 .
\end{aligned}
$$

The last equality comes from $\frac{\partial}{\partial \tau} \mathcal{P}_{\sigma}(\lambda, \tau)+$ $\mathcal{P}_{\sigma}(\lambda, \tau) \operatorname{He}\left(\Gamma_{\lambda}\right)=0$.

Let us proceed now to analyze the second stability condition from [33, Theorem 1]. To do so, we take into account the special structure of hybrid system (8)-(11) which implies that the jumps occur at the ordinary time instants either $t_{j}=\left(\frac{j}{2}+\lambda_{j}\right) T_{p}$ for even $j$ or $t_{j}=\frac{j+1}{2} T_{p}$ for odd $j$. We adopt here the following notation according to the hybrid time domain (12): $x_{j}=x\left(t_{j}, j\right), \lambda_{j}=\lambda\left(t_{j}, j\right)$, $\tau_{j}=\tau\left(t_{j}, j\right)$ that correspond to the variables right before the jump at $t_{j} \in\left\{\left(\frac{j}{2}+\lambda_{j}\right) T_{p},\left(\frac{j+1}{2}+\lambda_{j}\right) T_{p}\right\}$ and $x_{j}^{+}=$ $x\left(t_{j+1}, j+1\right), \lambda_{j}^{+}=\lambda\left(t_{j+1}, j+1\right)$ and $\tau_{j}^{+}=\tau\left(t_{j+1}, j+1\right)$ right after the same jump. In the same way, we define $\Delta V=V\left(x_{j}^{+}, \lambda_{j}^{+}, \tau_{j}^{+}\right)-V\left(x_{j}, \lambda_{j}, \tau_{j}\right)$. Note that $x_{j}^{+}=x_{j}$, $\left(\lambda_{j}^{+}, \lambda_{j}, \tau_{j}^{+}, \tau_{j}\right) \in\left\{\left(\lambda_{j}, \lambda_{j}, \lambda_{j} T_{p}, \lambda_{j} T_{p}\right),\left(\lambda_{j}^{+}, \lambda_{j}, 0, T_{p}\right)\right\}$.

There are two cases where the solution is in $\mathcal{D} \backslash \mathcal{A}$ :
- $\left(\lambda_{j}^{+}, \lambda_{j}, \tau_{j}^{+}, \tau_{j}\right)=\left(\lambda_{j}, \lambda_{j}, \lambda_{j} T_{p}, \lambda_{j} T_{p}\right):$ Here, we have

$$
\begin{aligned}
& \Delta V=W\left(x_{j}, \lambda, 1, \lambda T_{p}\right)-W\left(x_{j}, \lambda, 0, T_{p}\right) \\
& =\left[\begin{array}{c}
x_{j} \\
1
\end{array}\right]^{\top} e^{-\Gamma_{0}^{\top} \lambda T_{p}}(\bar{P}-\bar{P}) P e^{-\Gamma_{0} \lambda T_{p}}\left[\begin{array}{c}
x_{j} \\
1
\end{array}\right]=0 .
\end{aligned}
$$

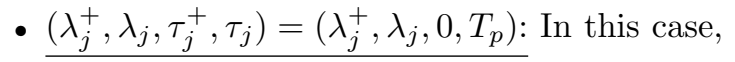

$$
\begin{aligned}
& \Delta V=W\left(x_{j}, \lambda_{j}^{+}, 0,0\right)-W\left(x_{j}, \lambda_{j}, 1, T_{p}\right) \\
& =\left[\begin{array}{c}
x_{j} \\
1
\end{array}\right]^{\top}\left(\bar{P}-\Psi\left(\lambda_{j}\right)^{\top} \bar{P} \Psi\left(\lambda_{j}\right)\right)\left[\begin{array}{c}
x_{j} \\
1
\end{array}\right] \\
& =\left[\begin{array}{c}
x_{j}^{+} \\
1
\end{array}\right]^{\top}\left(\bar{P}-\Psi\left(\lambda_{j}^{+}\right)^{\top} \bar{P} \Psi\left(\lambda_{j}^{+}\right)\right)\left[\begin{array}{c}
x_{j}^{+} \\
1
\end{array}\right]
\end{aligned}
$$

where $\Psi\left(\lambda_{j}\right):=e^{\Gamma_{1}\left(\lambda_{j} T_{p}-T_{p}\right)} e^{-\Gamma_{0} \lambda_{j} T_{p}}$.

Notice that the manipulable signal, $\lambda_{j+1}=\lambda_{j}^{+}$has to be computed at $t_{j}=\frac{j+1}{2} T_{p}$. For this, it is convenient to write $\Delta V$ in terms of $x_{j}$ instead of $x_{j+1}$. Hence, from (13), we obtain the following relationship

$$
\left[\begin{array}{c}
x_{j}^{+} \\
1
\end{array}\right]=\Psi\left(\lambda_{j}^{+}\right)\left[\begin{array}{c}
x_{j} \\
1
\end{array}\right] .
$$

We have

$$
\begin{aligned}
\Delta V & =\left[\begin{array}{c}
x_{j} \\
1
\end{array}\right] \bar{\Psi}\left(\lambda_{j}^{+}\right)^{\top}\left(\bar{P}-\Psi\left(\lambda_{j}^{+}\right)^{\top} \bar{P} \Psi\left(\lambda_{j}^{+}\right)\right) \bar{\Psi}\left(\lambda_{j}^{+}\right)\left[\begin{array}{c}
x_{j} \\
1
\end{array}\right] \\
& =\left[\begin{array}{c}
x_{j} \\
1
\end{array}\right]^{\top}\left(\bar{\Psi}\left(\lambda_{j}^{+}\right)^{\top} \bar{P} \bar{\Psi}\left(\lambda_{j}^{+}\right)-\bar{P}\right)\left[\begin{array}{c}
x_{j} \\
1
\end{array}\right],
\end{aligned}
$$

where $\bar{\Psi}\left(\lambda_{j}^{+}\right):=\Psi\left(\lambda_{j}^{+}\right)^{-1}$, which has been adopted for readability. It is worth to highlight that $\lambda_{j}^{+}$, which depends on $x_{j}$ according to the definition of $\mathcal{H}$, is associated with the value of $x$ at the jump instants. Indeed, $\left(x_{j}, \lambda_{j}^{+}, 0,0\right)$ refers here to the initial value in each hybrid arc. In the sequel, with abuse of notation we will use $\lambda$ and $x$ to represent $\lambda_{j}^{+}$and $x_{j}$, respectively.

In order to analyze the sign of (23), we must focus on small but nonzero values of the sampling period. With that aim, we will start by making explicit the dependence of this expression on $T_{p}$, for which the following decomposition is introduced.

$$
\begin{aligned}
& \Delta V=\left[\begin{array}{c}
x \\
1
\end{array}\right]^{\top}\left(\bar{\Psi}\left(\lambda_{j}^{+}\right)^{\top} \bar{P} \bar{\Psi}\left(\lambda_{j}^{+}\right)-\bar{P}\right)\left[\begin{array}{l}
x \\
1
\end{array}\right] \\
& =\left[\begin{array}{c}
x \\
1
\end{array}\right]\left[\begin{array}{cc}
\operatorname{He}\left(P A_{\lambda^{+}}\right) & \left(P B_{\lambda^{+}}\right) \\
\left(B_{\lambda^{+}}^{\top} P\right) & 0
\end{array}\right]\left[\begin{array}{l}
x \\
1
\end{array}\right] T_{p}+\mathcal{O}\left(T_{p}^{2}\right)
\end{aligned}
$$

with $A_{\lambda^{+}}:=A_{1}+\left(A_{0}-A_{1}\right) \lambda^{+}$and $B_{\lambda^{+}}:=B_{1}+\left(B_{0}-\right.$ $\left.B_{1}\right) \lambda^{+}$, after dropping subscript $j$ for ease of notation. The term $\mathcal{O}\left(T_{p}^{2}\right)$ is the residue of the first order MacLaurin series expansion on $T_{p}$. Now, let us assume that the maximum eigenvalue of the quadratic form of the residue 
is upper bounded by a function of the design constants $\alpha_{2}$ and $\alpha_{1}$, as follows,

$$
\lambda_{\max }\left(\frac{d^{2}\left(\bar{\Psi}\left(\lambda^{+}, v\right)^{\top} \bar{P} \bar{\Psi}\left(\lambda^{+}, v\right)\right)}{2 d v^{2}}\right) T_{p} \leq \min \left(\alpha_{1}, \alpha_{2}\right)
$$

$$
\forall \lambda^{+} \in[0,1], \quad v \in\left[0, T_{p}\right] .
$$

Note that $v$ is the uncertain variable of the Lagrange remainder of the MacLaurin expansion, and takes the role of $T_{p}$ in the $\bar{\Psi}$ function definition. Note also that the the maximum value of $T_{p}$ satisfying this condition is the maximum sampling time $T_{p}^{*}$ of Theorem 1. Eq. (25) would immediately imply that

$\Delta V \leq\left[\begin{array}{c}x \\ 1\end{array}\right]\left[\begin{array}{cc}\operatorname{He}\left(P A_{\lambda^{+}}\right) & \left(P B_{\lambda^{+}}\right) \\ \left(B_{\lambda^{+}}^{\top} P\right) & 0\end{array}\right]\left[\begin{array}{c}x \\ 1\end{array}\right] T_{p}+\left(\alpha_{1}+\alpha_{2}\|x\|^{2}\right) T_{p}$

which will be used along the rest of the proof. It will also be shown that these constants determine the size of the domain of attraction, which highlights a tradeoff between $T_{p}^{*}$ and the size of the convergence set.

Now, assuming that (25) holds, dividing (24) by $T_{p}$ and substituting the upper bound of $\mathcal{O}\left(T_{p}^{2}\right)$ where required, gives,

$$
\begin{aligned}
\frac{\Delta V}{T_{p}} & \leq x^{\top} \operatorname{He}\left(A_{\lambda^{+}}^{\top} P+\alpha_{2} I\right) x+2 B_{\lambda^{+}}^{\top} P x+\alpha_{1} \\
& =x^{\top} \operatorname{He}\left(A_{\lambda^{+}}^{\top} P+\alpha_{2} I\right) x+2\left(B_{1}^{\top}+\lambda^{+}\left(B_{0}-B_{1}\right)^{\top}\right) P+\alpha_{1} \\
& =x^{\top} \operatorname{He}\left(A_{\lambda^{+}}^{\top} P+\alpha_{2} I\right) x+2\left(1-\frac{\lambda^{+}}{\lambda_{e}}\right) B_{1}^{\top} P x+\alpha_{1} .
\end{aligned}
$$

The last step has been obtained by applying the property

$$
B_{1}+\left(B_{0}-B_{1}\right) \lambda_{e}=0 \Rightarrow B_{0}=-\frac{1-\lambda_{e}}{\lambda_{e}} B_{1},
$$

stemmed from Assumption 1 and the error equation (3). Remember that $\lambda^{+} \in[0,1]$. First, consider that $B_{1}^{\top} P x=$ 0 , then

$$
\begin{aligned}
\frac{\Delta V}{T_{p}} & \leq x^{\top} \operatorname{He}\left(A_{\lambda^{+}}^{\top} P+\alpha_{2} I\right) x+\alpha_{1}<-x^{\top} Q x+\alpha_{1} \\
& <-x^{\top} P x+\alpha_{1}<0
\end{aligned}
$$

applying LMIs (18)-(17) and $Q \succ P$. Then, (29) is negative for all $\xi \in \mathcal{D}_{1} \backslash \mathcal{A}$.

Now, we take into account $B_{1}^{\top} P x \neq 0$ and we distinguish 3 cases:

- $0<\lambda^{+}<1$ : Inserting (19) in (27), applying conditions LMI (18)-(17), $M \prec Q-P$ and assuming that $\lambda^{+}$is not saturated in (27), yields

$$
\begin{aligned}
\frac{\Delta V}{T_{p}} & \leq x^{\top} \operatorname{He}\left(A_{\lambda^{+}}^{\top} P+\alpha_{2} I\right) x+x^{\top} M x+\alpha_{1} \\
& <-x^{\top}(Q-M) x+\alpha_{1} \\
& <-x^{\top} P x+\alpha_{1}<0 \quad \forall \xi \in \mathcal{D}_{1} \backslash \mathcal{A} .
\end{aligned}
$$

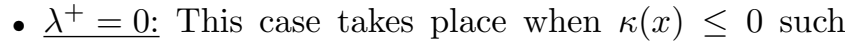
that (27) becomes

$$
\frac{\Delta V}{T_{p}}=x^{\top} \operatorname{He}\left(A_{0}^{\top} P+\alpha_{2} I\right) x+2 B_{1}^{\top} P x+\alpha_{1} .
$$

There are two possibilities, either $0 \preceq M \preceq Q$ or $M \preceq$ 0 . First, let us consider $M \preceq 0$. Here, the saturation in $\lambda^{+}=0$ is reached if $B_{1}^{\top} P x<0$ (necessary for the argument of (19) to be negative or zero), being (30) negative for all $\xi \in \mathcal{D}_{1} \backslash \mathcal{A}$ from the fact that condition (18) is satisfied.

Secondly, if $0 \preceq M \preceq Q$, then

$$
\lambda_{e}\left(1-\frac{x^{\top} M x}{2 B_{1}^{\top} P x}\right) \leq 0 \Rightarrow 2 B_{1}^{\top} P x \leq x^{\top} M x
$$

which implies, from (18),

$$
\begin{aligned}
\frac{\Delta V}{T_{p}} & \leq x^{\top} \operatorname{He}\left(A_{0}^{\top} P+\alpha_{2} I\right) x+x^{\top} M x+\alpha_{1} \\
& <-x^{\top}(Q-M) x+\alpha_{1} .
\end{aligned}
$$

Consequently, from $M \prec Q-P$ the following holds

$$
\frac{\Delta V}{T_{p}} \leq-x^{\top} P x+\alpha_{1}<0 \quad \forall \xi \in \mathcal{D}_{1} \backslash \mathcal{A} .
$$

- $\underline{\lambda}^{+}=1$ : In this case, corresponding to the case when $\kappa(x)$ is saturated in its upper bound, making (27) equal to

$$
\frac{\Delta V}{T_{p}}=x^{\top} \operatorname{He}\left(A_{1}^{\top} P+\alpha_{2} I\right) x+2 B_{0}^{\top} P x+\alpha_{1} .
$$

Once again, two situations must be considered, $0 \preceq$ $M \preceq Q$ and $M \preceq 0$. The saturation of the expression of $\kappa(x)$ at $\lambda^{+}=1$ with $M \preceq 0$ can only happen if $B_{1}^{\top} P x>0$, which implies, from the equilibrium equation (28), that

$$
2 B_{0}^{\top} P x=-2 \frac{1-\lambda_{e}}{\lambda_{e}} B_{1}^{\top} P x<0 .
$$

Therefore, (31) is negative for all $\xi \in \mathcal{D}_{1} \backslash \mathcal{A}$, if (17) is satisfied. Noting that $0 \preceq M \preceq Q$ and

$$
\lambda_{e}\left(1-\frac{x^{\top} M x}{2 B_{1}^{\top} P x}\right) \geq 1
$$

we obtain the following condition

$$
x^{\top} M x \geq-2 \frac{1-\lambda_{e}}{\lambda_{e}} B_{1}^{\top} P x=2 B_{0}^{\top} P x .
$$

The last step stems from (28). Then, taking the same steps as in the case $0<\lambda^{+}<1$, yields

$$
\frac{\Delta V}{T_{p}}<-x^{\top}(Q-M) x+\alpha_{1}<-x^{\top} P x+\alpha_{1} .
$$

Hence, for $\kappa(x)$ saturated in $\lambda^{+}=1$, we obtain

$$
\frac{\Delta V}{T_{p}}<0 \quad \forall \xi \in \mathcal{D}_{1} \backslash \mathcal{A} .
$$

Consequently, the solution at jump instants verify that $\frac{\Delta V}{T_{p}}<0 \forall(x, \lambda, \sigma, \tau) \in \mathcal{D}_{1} \backslash \mathcal{A} \subset \mathcal{D}$, which ensures that set $\mathcal{A}^{p}$ is attractive for small enough values of $T_{p}$. 
The last step is to prove that $\mathcal{A}$ is an invariant set, i.e., $g\left(\mathcal{A} \cap \mathcal{D}_{1}\right) \subset \mathcal{A}$ (remember that $W$ does not change when $\left.(x, \lambda, \sigma, \tau) \in \mathcal{C} \cup \mathcal{D}_{0}\right)$. To do so, remember that we obtained

$$
W\left(x, \lambda^{+}, 0,0\right)-W\left(x, \lambda, 1, T_{p}\right)<-x^{\top} P x+\alpha_{1} .
$$

Moreover, note that $W\left(x, \lambda^{+}, 0,0\right)=x^{\top} P x$. Then, after some manipulations, we get

$$
W\left(x, \lambda^{+}, 0,0\right)-\alpha_{1}<\frac{1}{2}\left(W\left(x, \lambda, 1, T_{p}\right)-\alpha_{1}\right) .
$$

Therefore, $W\left(x, \lambda^{+}, 0,0\right)-\alpha_{1}$ is negative in the jumps for any $(x, \lambda, \sigma, \tau) \in \mathcal{A}$. Hence, if the solution to $\mathcal{H}$ reaches $\mathcal{A}$, it will remain therein.

Finally, applying the nonsmooth invariance principle given in [33], and using the well posedness result established at the beginning of the proof, we can conclude that, for small enough values of $T_{p}, \mathcal{A}$ is UGAS.

Proof of (ii): Now, we desire to prove that $\mathcal{L} \subset \mathcal{A}$. This is direct noting that $V(\xi)$ is only updated in each $x\left(t_{2 k}\right)$ of (4)-(5) that means when $x$ is in $\mathcal{A} \cap \mathcal{D}_{1}$. Therefore, $W(\xi)$ takes the same value for each couple $\left(x\left(t_{2 k-1}\right), x\left(t_{2 k}\right)\right)$, ensuring that $x_{c}:=x_{e, T_{p}}$ is inside $\mathcal{A}$.

Remark 1: It is worth noting that the maximal solutions to $\mathcal{H}$ with (19) are complete. This property comes from applying [14, Prop. 6.10]. Indeed, the flow set $\mathcal{C}$ is the closure of the complement of the jump set $\mathcal{D}$. In other words, $\mathcal{C} \cup \mathcal{D}$ covers the complete state space and the boundary of $\mathcal{C}$ belongs to $\mathcal{D}$. Moreover, item (c) never happens because $G(\mathcal{D}) \subset \mathcal{C} \cup \mathcal{D}$ and if Theorem 1 conditions are satisfied, then the solutions remain bounded avoiding the possibility of item (b). Then, we can conclude that the maximal solutions are complete, because the only possibly is item (a).

Remark 2: It is worth noting that the particular case $M=0$ means an open-loop control with

$$
\kappa(x)=\lambda_{e}
$$

Indeed, Theorem (1) proves UGAS property of the attractor even with this particular control law. Nevertheless, the open-loop character of this case makes this control law unsuitable for practical applications.

Remark 3: The maximum amplitude of the chattering in steady state for each variable of $x$ is defined by $\max \left\{x_{e, T_{p}}, x_{e, \lambda_{e T_{p}}}\right\}$.

\section{A. Adjusting $P$}

Theorem 1 requires that some conditions for matrices $P, Q$ and $M$ are satisfied, leading to a feasible problem with many solutions for these matrices. However, we are interesting in reducing the volume of $\mathcal{A}$ to bound the limit cycle $\mathcal{L}$. To this end, we formulate the following convex optimization problem which present feasible solutions

$$
\begin{aligned}
\min _{P, Q} \quad- & \log (\operatorname{det}(P)) \\
& A_{0}^{\top} P+P A_{0}+\alpha_{2} I \prec-Q \\
& A_{1}^{\top} P+P A_{1}+\alpha_{2} I \prec-Q . \\
& P \prec Q \\
& M \prec Q-P .
\end{aligned}
$$

\section{B. Adjusting $M$}

This tuning parameter adjusts the transient time, modifying the response time, voltage oscillations, current peak, among others. Indeed, if $M \preceq 0$, the system response can be faster and/or can present current peaks and voltage oscillations, with tendency to saturate the control signal. Conversely, if $0 \preceq M \preceq Q$, the system can reduces the oscillations, diminishing the current peaks.

\section{Simulations}

Some simulations are performed to validate the results proposed here. For this, we select a boost converter whose model matrices are [34]:

$$
\begin{gathered}
A_{1}=\left[\begin{array}{cc}
-R / L & 0 \\
0 & -1 / R_{0} C_{0}
\end{array}\right], \quad A_{2}=\left[\begin{array}{cc}
-R / L & -1 / L \\
1 / C_{0} & -1 / R_{0} C_{0}
\end{array}\right], \\
B_{1}=B_{2}=\left[\begin{array}{c}
V_{\text {in }} / L \\
0
\end{array}\right],
\end{gathered}
$$

where the physical parameters are given in Table I. The physical state vector is $z=\left[\begin{array}{ll}i_{L} & v_{C}\end{array}\right]$, where $i_{L}$ and $v_{C}$ are the inductor current and capacitor voltage, respectively.

TABLE I

BOOST CONVERTER PARAMETERS.

\begin{tabular}{ll} 
COMPONENT & NOMINAL VALUE \\
\hline$V_{i n}$ & $24 \mathrm{~V}$ \\
$R$ & $11.5 \mathrm{~m} \Omega$ \\
$L$ & $470 \mu \mathrm{H}$ \\
$C$ & $20 \mu \mathrm{F}$ \\
$R_{0}$ & $50 \Omega$ \\
\hline
\end{tabular}

The selected operating point is $z_{e}=\left[\begin{array}{ll}8.4 & 100\end{array}\right]^{T}$, which is associated with $\lambda_{e}=0.76$.

The optimization problem (33) provides

$$
Q=\left[\begin{array}{cc}
6.12 & 0 \\
0 & 1.35
\end{array}\right] \cdot 10^{7}, \quad P=\left[\begin{array}{cc}
1.58 & 0 \\
0 & 0.67
\end{array}\right] \cdot 10^{5} .
$$

Moreover, $\alpha_{1}=1.68 \cdot 10^{4}$ and $\alpha_{2}=8.58 \cdot 10^{5}$. For ease of implementation and without performance losses, we have relaxed condition (25), by using a less conservative value $T_{p}=10 \mu s$.

Figure 5 shows a zoom of the state variables, the control input $\sigma$ and the Lyapunov function $V(\xi)$ in transient time. It can be observed that the Lyapunov function is constant, excepts at time instants $t=k T_{p}$ with $k \in \mathbb{N}$, when it jumps a step downwards. Moreover, the control input $\sigma$ evolves by switching between the functioning modes $\sigma \in\{0,1\}$. 

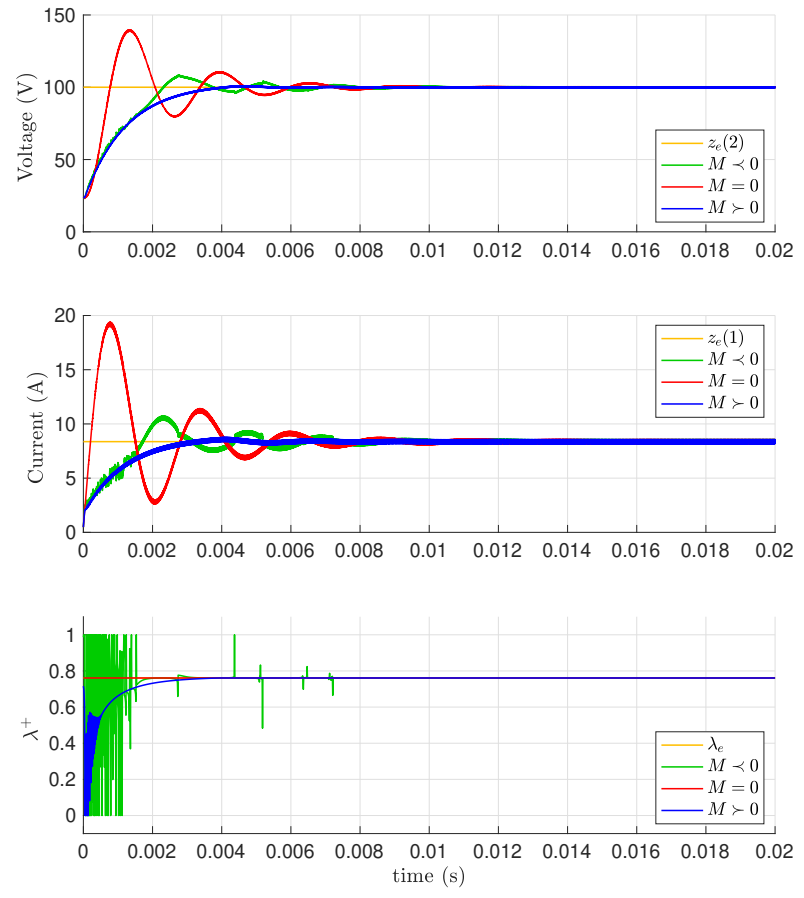

Fig. 4. State and control input $\left(\lambda^{+}\right)$evolutions, with $M \prec 0$, in green, $M=0$, in red, and $M \succ 0$, in blue. The reference is shown in yellow.

TABLE II

Circuit parameters values

\begin{tabular}{lll} 
COMPONENT & VAlue & Model \\
\hline$V_{I N}$ & $24 \mathrm{~V}$ & \\
$L$ & $470 \mu \mathrm{H}$ & AGP4233-474ME \\
$R$ & $11.5 \mathrm{~m} \Omega$ & \\
$C_{1}$ & $20 \mu \mathrm{F}$ & MKP1848C62090JP4 \\
$R_{C}$ & $5 \mathrm{~m} \Omega$ & \\
$R_{0}$ & $50 \Omega$ & \\
Diode & & C3D06060A \\
switch & & C3M0065090D \\
Driver & & 1EDI20N12AF \\
\hline
\end{tabular}

\section{EXPERIMENTAL SETUP}

A test setup was built to validate the proposed hybrid scheme. Fig. 6 shows this experimental set up. It is composed of:

- A boost converter whose electrical parameters are given in Table II. These parameters correspond to the ones used in the simulations of Sect. V.

- An electronic card (model LEM LTS 15-NP) for the measurements of the inductor current and voltage sensor for the measurement of the output voltage. We built the voltage sensor by means of a resistor divider connected with an operational amplifier in buffer configuration.

- A dSPACE cardboard (DS1103) with a PowerPC 604e at $400 \mathrm{MHz}$ and a fixed-point DSP TMS320F240.

The complete control scheme was implemented in the dSPACE card by means of Matlab-Simulink.
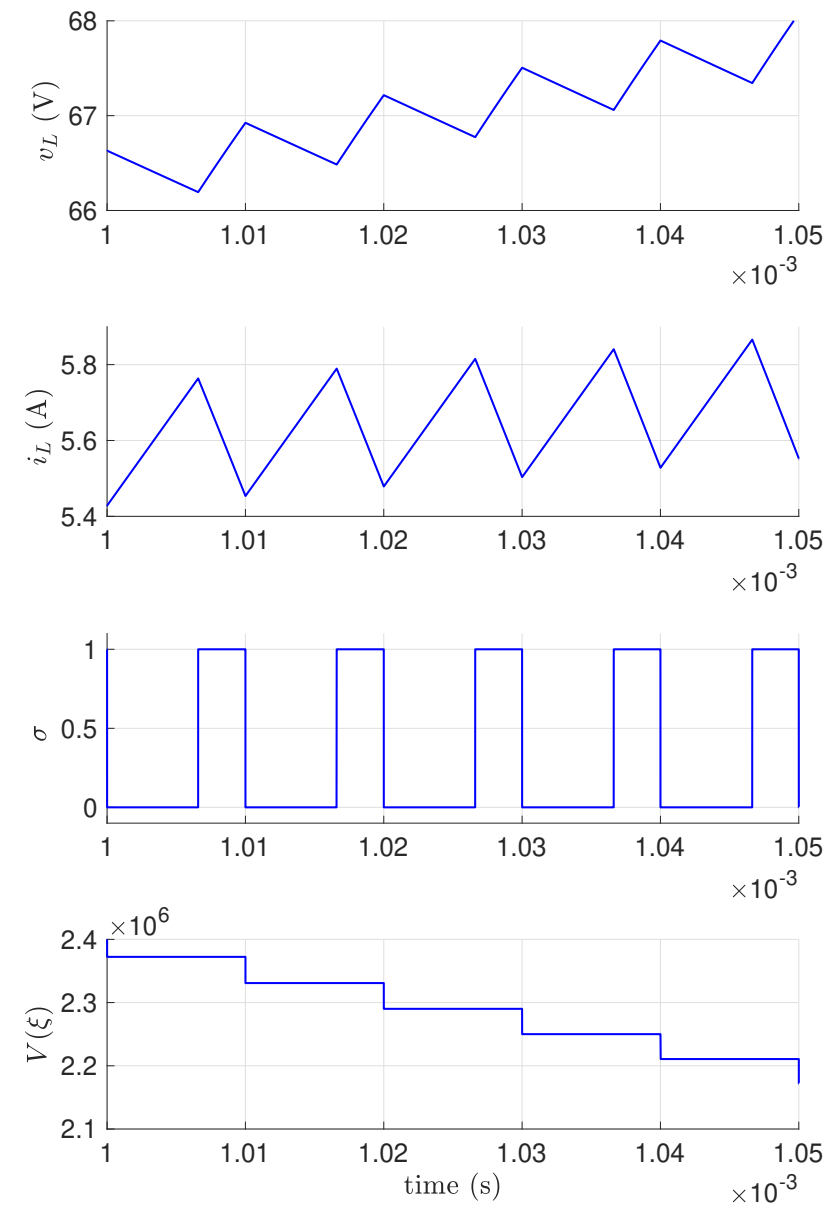

Fig. 5. Zoom of the state, control input $(\sigma)$ and Lyapunov function evolutions, with $M \succ 0$ in transient time.

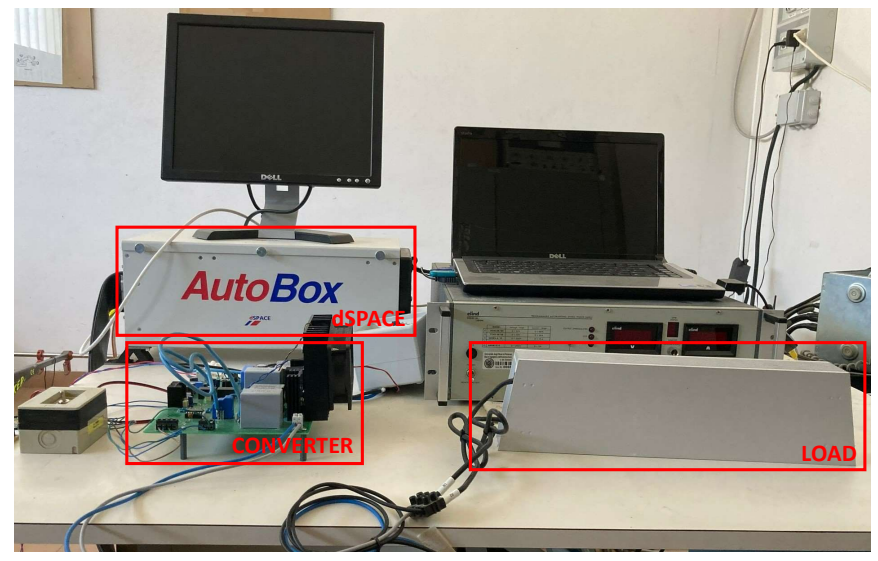

Fig. 6. Experimental set up. 

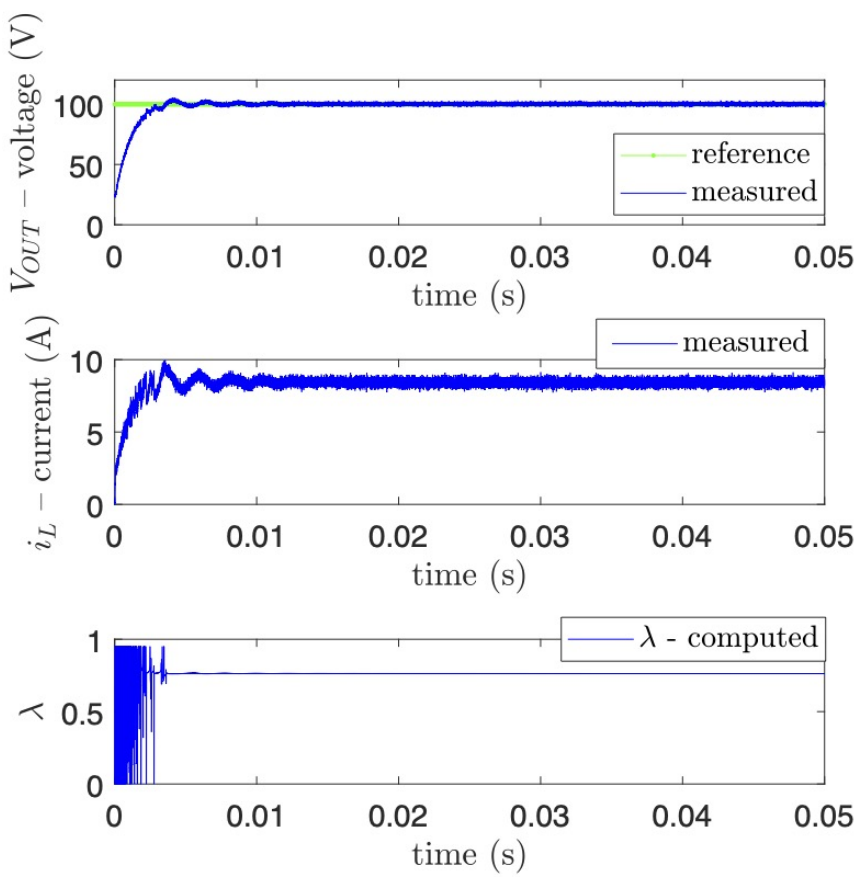

Fig. 7. Evolutions of the voltage, current and duty cycle in the start up.

We selected for these tests $M=0.1 Q$, corresponding to the best results obtained in Sect V.

Fig. 7 shows a start up transient from a initial condition equal to $x_{0}=\left[\begin{array}{ll}0 & V_{I N}\end{array}\right]$ to a reference operating point computed by imposing an output voltage equal to $V_{\text {out }}=100 \mathrm{~V}$. The voltage and the current signals present a smooth behavior, as performed in simulation (see Fig. 4). Likewise, the steady state operation is shown in Fig. 8.

Finally, a perturbation in the load was introduced. The load $R_{0}$ was changed from $R_{0}=50 \Omega$ to $R_{0}=75 \Omega$ at $0.02 \mathrm{~s}$. The proposed algorithm does not provide a voltage output regulation, and an error in the voltage output is exhibited in steady state. However, if an external loop is added with a PI control as in [35], the output voltage is regulated in its reference value, maintaining a good performance.

\section{Conclusion}

A hybrid model of switched power converters with PWM inputs and a sample-and-hold mechanism is proposed here. The study is particularized for two functioning modes, which is a common power converter application. Moreover, a new control algorithm that chooses the value of the duty cycle at the beginning of each PWM-sampling interval is designed from a rigorous model. For this choice of controller, HDS stability analysis is done, proving convergence to a limit set that can be bounded based on the choice of the size of the sampling period. The hybrid analysis also provides a Lyapunov function for the closed-loop system. Finally, an upper and lower bound for the chattering peaks obtained by the signals is provided. Experimental results show satisfactory closed-loop performance.

PWMs with different carriers and converters with more than 2 modes will be considered in future works. Moreover,
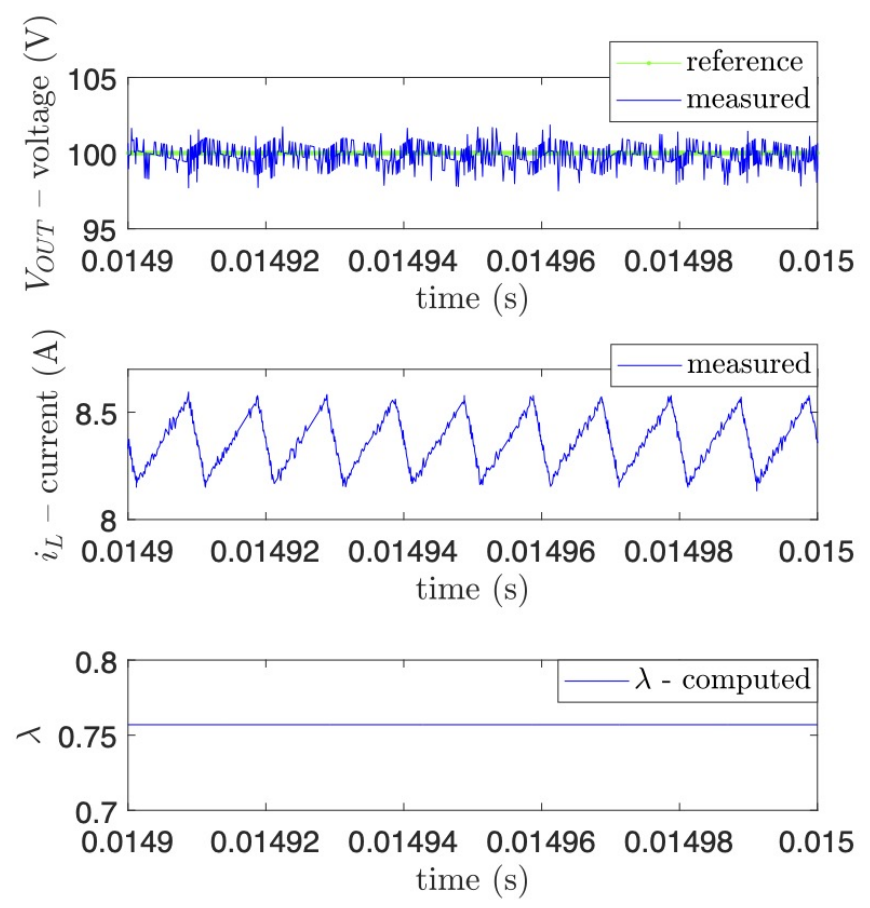

Fig. 8. Evolutions of the voltage, current and duty cycle in the steady state.
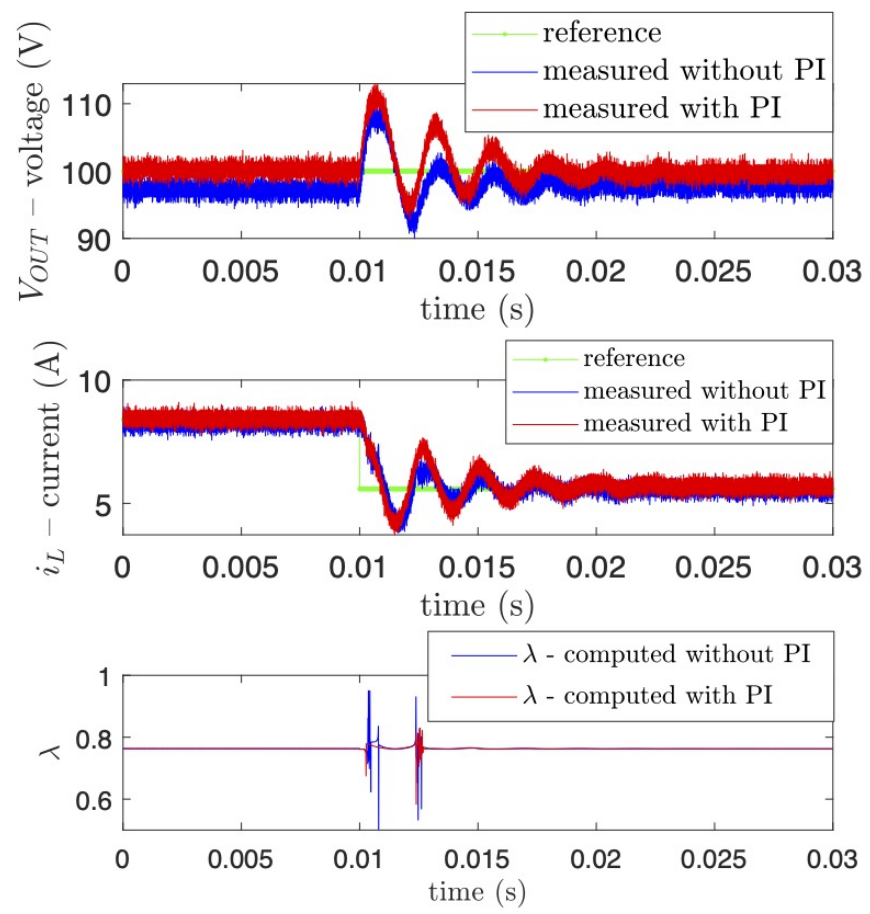

Fig. 9. Evolutions of the voltage, current and duty cycle without PI controller (in red) and with PI controller (in blue) for regulating the voltage output with a perturbation of $R_{0}$. 
a stability analysis with an external control loop that control the output voltage is expected. The consideration of more switches is left for future research.

\section{REFERENCES}

[1] H. Kanaan, Kamal Al-Haddad, and F. Fnaiech. Modelling and control of three-phase/switch/level fixed-frequency PWM rectifier: state-space averaged model. IEE Proceedings-Electric Power Applications, 152(3):551-557, 2005.

[2] A. Yazdani and R. Iravani. A generalized state-space averaged model of the three-level NPC converter for systematic DCvoltage-balancer and current-controller design. IEEE Trans. on Power Delivery, 20(2):1105-1114, 2005.

[3] PG Handley and JT Boys. Practical real-time PWM modulators: an assessment. In IEE Proceedings $B$ (Electric Power Applications), volume 139, pages 96-102. IET, 1992.

[4] S. K. Peddapelli. Pulse Width Modulation. Other Titles in Applied Mathematics. De Gruyter Oldenbourg, 2016.

[5] Omar Ali Beg, Houssam Abbas, Taylor T. Johnson, and Ali Davoudi. Model validation of PWM DC-DC converters. IEEE Trans. on Industrial Electronics, 64(9):7049-7059, 2017.

[6] M.B. D'Amico, N. Guerin, A. Oliva, and E.E. Paolini. Dinámica de un convertidor buck con controlador PI digital. Revista Iberoamericana de Automática e Informática Industrial RIAI, 4(3):126-131, 2007.

[7] D. J. Perreault and G. C. Verghese. Time-varying effects and averaging issues in models for current-mode control. IEEE Trans. on Power Electronics, 12(3):453-461, 1997.

[8] L. Iannelli, Karl H.J., Ulf T. Jönsson, and F. Vasca. Subtleties in the averaging of a class of hybrid systems with applications to power converters. Control Engineering Practice, 16(8):961-975, aug 2008.

[9] D.G. Holmes and T.A. Lipo. Pulse width modulation for power converters: principles and practice, volume 18. John Wiley \& Sons, 2003.

[10] E. Vidal-Idiarte, A. Marcos-Pastor, G. Garcia, A. Cid-Pastor, and L. Martinez-Salamero. Discrete-time sliding-mode-based digital pulse width modulation control of a boost converter. IET Power Electronics, 8(5):708-714, 2015.

[11] P. Cortés, M.P. Kazmierkowski, R.M. Kennel, D.E. Quevedo, and J. Rodríguez. Predictive control in power electronics and drives. IEEE Trans. on industrial electronics, 55(12):4312-4324, 2008.

[12] F.M. Oettmeier, J. Neely, S. Pekarek, R. DeCarlo, and K. Uthaichana. MPC of switching in a boost converter using a hybrid state model with a sliding mode observer. IEEE Trans on Industrial Electronics, 9(56):3453-3466, 2009.

[13] S. Baldi, A. Papachristodoulou, and E. B. Kosmatopoulos. Adaptive pulse width modulation design for power converters based on affine switched systems. Nonlinear Analysis: Hybrid Systems, 30:306-322, 2018.

[14] R. Goebel and R.G. Sanfelice and A.R. Teel. Hybrid Dynamical Systems: modeling, stability, and robustness. Princeton University Press, 2012

[15] R. A. Decarlo, M. S. Branicky, S. Pettersson, and B. Lennartson. Perspectives and results on the stability and stabilizability of hybrid systems. Proceedings of the IEEE, 88(7):1069-1082, 2000.

[16] D. Liberzon and A. S. Morse. Basic problems in stability and design of switched systems. IEEE Control Systems Magazine, 19(5):59-70, 1999.

[17] D. Liberzon. Switching in Systems and Control. Other Titles in Applied Mathematics. Springer, 2003.

[18] R. Goebel, R. Sanfelice, and A.R. Teel. Hybrid dynamical systems. IEEE Control Systems Magazine, 29(2):28-93, April 2009.

[19] A.R. Teel and D. Nešić. PWM hybrid control systems: averaging tools for analysis and design. In IEEE International Conference on Control Applications, 2010.

[20] L. Hetel and E. Bernuau. Local stabilization of switched affine systems. IEEE Trans. on Automatic Control, 60(4):1158-1163, 2015.

[21] G. S. Deaecto and J. C. Geromel. Stability analysis and control design of discrete-time switched affine systems. IEEE Trans. on Automatic Control, 62(8):4058-4065, 2017.
[22] P. Hauroigne, P. Riedinger, and C. Iung. Switched affine systems using sampled-data controllers: Robust and guaranteed stabilization. IEEE Trans. on Automatic Control, 56(12):2929$2935,2011$.

[23] G. S. Deaecto, J. C. Geromel, F. S. Garcia, and J. A. Pomilio. Switched affine systems control design with application to DCDC converters. IET Control Theory Applications, 4(7):12011210,2010

[24] C. Albea-Sanchez, G. Garcia and L. Zaccarian. Hybrid dynamic modeling and control of switched affine systems: application to DC-DC converters. In 54th IEEE Conference on Decision and Control, Osaka, Japan, December 2015. An extended version is avaible in: https://hal.archivesouvertes.fr/hal-01220447v3/document.

[25] A. Sferlazza, C. Albea-Sanchez, L. Martínez-Salamero, G. Garcia, and C. Alonso. Min-type control strategy of a dc-dc synchronous boost converter. IEEE Trans. on Industrial Electronics, 67(4):3167-3179, 2019.

[26] C. Albea-Sanchez, G. Garcia, S. Hadjeras, W. P. M. H. Heemels, and L. Zaccarian. Practical stabilization of switched affine systems with dwell-time guarantees. IEEE Trans. on Automatic Control, 64(11):4811-4817, 2019.

[27] C. Albea Sanchez, O. Lopez Santos, D. Prada Zambrano, F. Gordillo, and G. Garcia. Hybrid control algorithm for a DC-AC converter. IEEE Trans. on Control System Technology, 2018.

[28] C. Albea-Sanchez and G. Garcia. Robust hybrid control law for a boost inverter. Control Engineering Practice, 101:104492, 2020.

[29] A. Sferlazza, C. Albea-Sanchez, and G. Garcia. A hybrid control strategy for quadratic boost converters with inductor currents estimation. Control Engineering Practice, 103:104602, 2020.

[30] D. Liberzon and A.S. Morse. Basic problems in stability and design of switched systems. IEEE control systems magazine, 19(5):59-70, 1999.

[31] G.S. Deaecto, J.C. Geromel, F.S. Garcia, and J.A. Pomilio. Switched affine systems control design with application to DCDC converters. IET control theory \& applications, 4(7):1201$1210,2010$.

[32] C. Albea and A. Seuret. Time-triggered and event-triggered control of switched affine systems via a hybrid dynamical approach. Nonlinear Analysis: Hybrid Systems, 41:101039, 2021.

[33] A. Seuret, C. Prieur, S. Tarbouriech, A.R. Teel, and L. Zaccarian. A nonsmooth hybrid invariance principle applied to robust event-triggered design. IEEE Trans. on Automatic Control, 64(5):2061-2068, 2018.

[34] C. Albea Sanchez, G. Garcia, and L. Zaccarian. Hybrid dynamic modeling and control of switched affine systems: application to DC-DC converters. In IEEE Conference on Decision and Control $(C D C), 2015$.

[35] A. Sferlazza, C. Albea Sanchez, L. Martinez-Salamero, G. Garcia, and C. Alonso. Min-type control strategy of a dcdc synchronous boost converter. IEEE Trans. on Industrial Electronics, 2019.

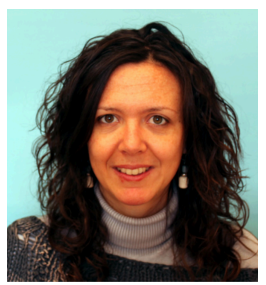

Carolina Albea received her Ph.D. in Automatic Control in 2010 from the University of Sevilla, Spain, and the University of Grenoble, France. From 2010 to 2011, she held a postdoctoral position at the CEA-LETI Minatec campus in Grenoble, France, on the control of nanoelectronic circuits. Then, from 2011 to 2020 she became Associate Professor at the University of Toulouse III (Université Paul Sabatier) and her research was performed at the LAAS- CNRS. Since 2020, she joined the University of Seville, Spain. Her topics of interest cover hybrid control of switched systems, hybrid dynamical systems application, distributed control, control of electronic converters. 


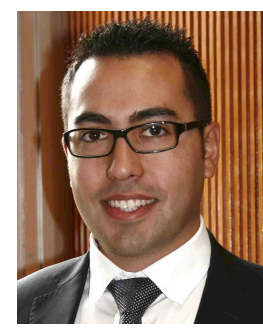

Antonino Sferlazza was born in Palermo, Italy, in 1987. He received the Master degree in automation engineering and the Ph.D. degree in mathematics and automation from the University of Palermo, Italy, in 2011 and 2015 respectively. In 2013 he was visiting PhD student at University of California at Santa Barbara, CA, USA. From 2016 to 2017 he join the University of Palermo as junior researcher. From 2017 to 2018 he was researcher are LAAS CNRS of Toulouse, France. He is currently a researcher in systems and control engineering at the University of Palermo. His research interests include the development of feedback control algorithms for nonlinear dynamical systems, estimation theory, and applications of control of electrical drives, power converters, and mechanical systems.

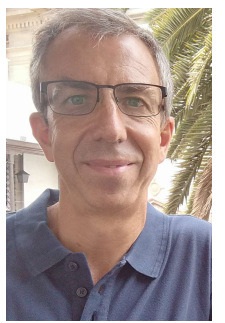

Francisco Gordillo received the M.Eng. and $\mathrm{Ph} . \mathrm{D}$. degrees in industrial engineering from the University of Seville, Seville, Spain, in 1988 and 1994, respectively. Since 1989, he has been with the Department of Automatic Control, Escuela Superior de Ingenieros, Universidad de Sevilla, where he is currently a Full Professor. He is the coauthor of Dinamica de Sistemas (Madrid: Alianza Editorial, 1997), coeditor of Stability Issues in Fuzzy Control (Berlin: Physica-Verlag, 2000), and author or coauthor of more than 150 publications including book chapters, journal articles, and conference proceedings. His research interests include nonlinear control with applications to mechanical, electromechanical, and power electronic systems.

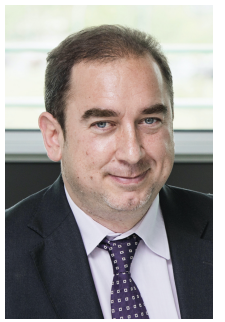

Fabio Gómez-Estern BSc+MSc in Telecommunications Engineering (University of Seville), $\mathrm{MSc}+\mathrm{PhD}$ in Control Systems (University of Seville), and BSc in Economics (University of London). He has worked in industry (Abengoa, France Telecom) and as a visiting researcher in several European laboratories. From 2002 to 2012 he has been Associate Professor at the University of Seville, where he has served as Head of Department of Automatic Control. He is currently Full Professor and Founding Director of the School of Engineering at the Universidad Loyola Andalucía in Seville, Spain. His research interest are: Nonlinear Control Systems, Distributed Control and Networked Control, with applications to Electromechanical Systems and power converters. 